\title{
From the Editor-in-Chief
}

\section{Shifting Shifts}

A press release of a recent study conducted by Rogers et al. (2004) of the University of Pennsylvania School of Nursing was picked up by newspapers, TV and radio. These investigators found that nurses who worked longer than 12 hours per shift committed more errors, as did nurses who worked overtime or more than 40 hours a week. Since most nurses regularly work overtime, these limits are routinely exceeded. The investigators recommended that nursing eliminate mandatory overtime and return to the eight-hour shift.

The nurse participants were randomly selected from members of the American Nurses Association, and this sample represents a 40\% response rate from those invited to participate. They were generally women (92\%), middle-aged (mean age 44.8), Caucasian (79\%) and experienced (mean years 17.2), traits that are representative of the American nursing population and similar to those of the average Canadian nurse. Nurses in the study kept a log over a two-week period (which involved a total of 5,317 shifts), noting the shift they were scheduled to work and did work; any overtime, days off, sleep/wake patterns; and their errors and near misses. Overtime was calculated when more than 12.5 and 8.5 hours were worked in scheduled 12- and eight-hour shifts, respectively. There are, as usual, limitations to the study. The sample size of 393 nurses is large but not huge, and the response rate is low $-\mathrm{a}$ factor that the authors attribute to the burden of participating.

There was a direct relationship between the length of the shifts and both the number of errors committed and the frequency of near misses. Nurses were three times more likely to commit errors when they worked more than 12.5 hours. "Working overtime increased the odds of making at least one error, regardless of how long the shift was originally scheduled" (Rogers et al. 2004: 204). The trend towards making errors accelerated with the length of the original shift - that is, your chance of committing an error was greater if you worked overtime after a 12-hour shift than after an eight-hour one.

This study purports to be the first to link overtime and shift lengths to patient safety; however, it refers to Canadian research that shows a link between 12-hour shifts and increased sick days, absenteeism and lower job satisfaction of nurses (Zboril-Benson 2002) and other work linking extended shifts with more accidents 
sustained by nurses. The authors of this study recommend that since more than three-quarters of scheduled 12-hour shifts extended beyond that time, routine use of 12-hour shifts should be limited, and overtime when 12-hour shifts are involved should be eliminated. Furthermore, all mandatory overtime should be eliminated.

... direct relationship between the length of the shifts and both the number of errors committed and the frequency of near misses. Nurses were three times more likely to commit errors when they worked more than 12.5 hours.
The Globe and Mail, a national Canadian newspaper, covered this story (July 8, 2004). The writer, André Picard, interviewed Linda Silas, president of the Canadian Federation of Nurses Unions, about the findings. Silas noted that 12 hour shifts would not likely disappear because nurses found them convenient; longer shifts required nurses to work fewer nights, gave them frequent

stretches of five days off and made it easier to arrange child care. Further, the advantages of 12-hour shifts offset to some extent the toll on lifestyle that nurses pay by regularly working evenings and nights, weekends and holidays. These trade-offs are not a small thing.

When I read the newspaper article and then the report of the study, I was struck by the challenge of trying to implement "best practice" when it comes to nurses' work life. I think most nurse administrators and clinical nurses have embraced using research to inform nursing practice. This includes giving up "beloved" practices honed over years to adopt practices identified through research that lead to better patient outcomes. However, research is also beginning to reveal best practices in administration and the structure of nurses' work life. The shiftwork study deserves the same response from administrators and clinical nurses as clinical research. What are the implications?

Clearly, a critical appraisal is needed of research into the effects of overtime and longer shifts on nurses' and patients' health and well-being. The study by Rogers et al. (2004) shows a link between overtime and patient safety - in this case, nurses' errors. But quality care goes beyond safety. What happens to the quality of nurse-patient relationships when nurses work overtime, especially towards the end of a 12-hour shift? What happens to nurses' ability to assess and manage patients' symptoms such as pain, dyspnea, nausea and fatigue when they are overfatigued themselves? Do patients receive the education they require to manage their health situations or achieve the same levels of functional ability when their nurses are regularly working overtime or long shifts? 
There is considerable research that shows nurses can and should make a difference to symptom management, patients' therapeutic self-care and functional ability (Doran 2003). If the critical appraisal demonstrates that we do not have research examining the relationship between overtime, length of shifts and patient outcomes, then that research must be undertaken.

Administrators cannot choose not to implement research findings about nurses' well-being and at the same time insist that nurses implement research findings about patient care. So, if research shows there is a link between poorer patient outcomes and nursing overtime, then nurse administrators have to find a solution to this problem. And if research shows that patients achieve better outcomes and are at lower risk for nursing errors when nurses work eight-hour shifts versus longer ones, then nurses must re-examine their attachment to 12-hour shifts despite their preference for them.

\section{References}

Doran, D., ed. 2003. Nurse-Sensitive Patient Outcomes: State of Science. Toronto: Jones and Bartlett.

Picard, A. 2004 (July 8). "Long Shifts Raise Risk of Nursing Errors.” The Globe and Mail: A13.

Rogezs, A.E., W. Hwang, L.D. Scott, L.H. Aiken and D.F. Dinges. 2004. “The Working Hours of Hospital Staff Nurses and Patient Safety." Health Affairs 23(4): 202-8.

Zboril-Benson, L.R. 2002. "Why Nurses Are Calling in Sick: The Impact of Health-Care Restructuring." Canadian Journal of Nursing Research 33(4): 89-107.

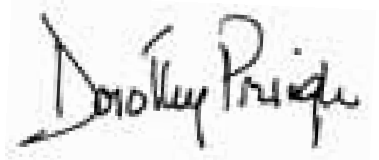

Dorothy Pringle, $\mathrm{PhD}$

Editor-in-Chief

\section{Study Links Long Hours, Nurse Errors}

What they've long known about truck drivers, airplane pilots and doctors, researchers also are discovering about nurses: Those who work more than 12 straight hours make more mistakes.

Nurses who worked shifts lasting at least 12.5 hours were three times more likely to commit an error, such as giving a patient the wrong medicine or the wrong dose, than nurses who worked less than 8.5 hours, about a regular shift, according to a new study from the University of Pennsylvania School of Nursing. 